\title{
BIBLIOGRAFIA
}

\section{Fuentes Bibliográficas para el Estudio de la Novela Chilena (1843-1960)}

El material bibliográfico que incluimos a continuación ha sido ordenado en cinco categorías que nos parecen de importancia para el estudio de la novela chilena. En la primera sección (Literatura chilena en general) se citan aquellos libros que ofrecen una breve visión panorámica del género como parte de la producción literaria total del país. La segunda sección incluye estudios particulares sobre tendencias determinadas tales como Romanticismo o Vanguardismo y sobre las actividades o ideología de una generación. En la siguiente categoría se ofrecen los estudios pertinentes a un escritor o a una novela. Debido a que varios críticos se han detenido a investigar un aspecto singular dentro de la trayectoria de la novela, hemos preferido agrupar estos estudios en una sección aparte que cubre los espacios, temas y motivos en la novela chilena. Finalmente, en la sección quinta, ofrecemos una lista de material bibliográfico sobre revistas literarias, tesis y crítica en general.

L.G.C.

\section{LITERATURA CHILENA EN GENERAL}

Alegría, Fernando. Las fronteras del Realismo. Santiago, Chile: Empresa Editora Zig Zag, 1962.

-... La literatura chilena del siglo XX. Santiago, Chile: Empresa Editora Zig Zag, 1962.

.... La literatura chilena contemporánea. Buenos Aires, Argentina: Centro Editor de América, 1968.

Amunátegui Solar, Domingo. Las letras chilenas. Santiago, Chile: Editorial Nascimento, 1934.

Cruz, Pedro Nolasco. Estudios sobre literatura chilena. Vol. I, Santiago, Chile: 
Zamorano y Caperán, 1926. Vols. II y III, Santiago, Chile: Editorial Nascimento, 1940.

Díaz Arrieta, Hernán [Alone]. Panorama de la literatura chilena durante el siglo $X X$. Santiago, Chile: Editorial Nascimento, 1931.

.... Historia personal de la literatura chilena. Santiago, Chile: Empresa Editora Zig Zag, 1954.

Dussuel, Francisco. Historia de la literatura chilena. Santiago, Chile: Ediciones Paulinas, 1959.

Latorre, Mariano. La literatura en Chile. Buenos Aires: Imprenta y Casa Editora Coni, 1941.

Lillo, Samuel. Literatura chilena. Santiago, Chile: Editorial Nascimento, 1952. Melfi, Domingo. Estudios de literatura chttena. Santiago, Chile: Editorial Nascimento, 1938.

-.... El viaje literario. Santiago, Chile: Editorial Nascimento, 1944.

Merino Reyes, Luis. Panorama de la literatura chilena. Washington, D.C.: Unión Panamericana, 1959.

Montes, Hugo, y Orlandi, Julio. Historia y antologia de la literatura chilena. Santiago, Chile: Editorial del Pacífico, 1955.

Rojas, Manuel. Manual de literatura chilena. México: Universidad Nacional Autónoma de México, 1964.

Silva Castro, Raúl. Panorama literario de Chile. Santiago, Chile: Editorial Universitaria, 1961.

Torres-Ríoseco, Arturo. Breve historia de la literatura chilena. México: Ediciones de Andrea, 1956.

Vaise, Emilio. Estudios criticos de literatura chilena. Santiago, Chile: Editorial Nascimento, 1940.

\section{MOVIMIENTOS LITERARIOS Y TENDENCIAS EN PARTICULAR}

Alegría, Fernando. "Orígenes del Romanticismo en Chile". Cuadernos Americanos, Año VI (sept-oct 1947), pp. 173-193.

.... "Sobre el encuentro de escritores en Concepción". Atenea, Año XXXV, CXXX, No. 379 (enero-marzo 1958), 165-178.

..... "Resolución de medio siglo". Atenea, Año XXXV, CXXXI NO. 380-381 (abr-sept 1958), 141-148.

Atías, Guillermo. "La literatura como lujo". Atenea, Año XXXV, CXXXI, No. 380-381 (abr-sept 1958), 49-58.

Austro. "La renovación literaria de 1900". Atenea, Año XIV, LVII, No. 170 (agosto 1939), 296-311.

Barros Arana, Diego. “El movimiento político de 1842”. Atenea, Año XVIII, 
LXVIII, No. 203 (may 1942), 269-289.

Bourgeois, Louis C. "The Tolstoy Colony: A Chilean Utopian-artistic Experiment”. Hispania, XLVI (1963), 514-518.

Campaña, Antonio. "Speaking of Chile: A Survey of Recent Cultural Trends". Américas, XIV (marzo 1962), 34-38.

Cassigoli, Armando. "Literatura y responsabilidad". Atenea, Año XXXV, CXXXI, No. 380-381 (abr-sept 1958), 58-66.

Cerda Cuitiño, Mario. "Santiago literario de mil novecientos". Estudios Filológicos, No. 2 (1966), pp. 41-61.

Costa, René de. "Recapitulación de la historia de Los Diez". Atenea, Año XLV, CLXVII, No. 420 (abr-jun 1968), pp. 11-27.

Crowley, Cornelius. "Costumbrism in Chilean Literary Prose of the Nineteenth Century". Disertación, University of California, Berkeley, 1944.

Chacón, Jacinto. "Una carta sobre los hombres de 1842". Atenea, Año XVIII, LXVIII, No. 203 (mayo 1942), 193-201.

Dávila Silva, Ricardo. Estado actual de la literatura chilena. Santiago, Chile: Imprenta Universitaria, 1924.

Donoso, Armando. Los nuevos. Valencia, España: Sempere y Cía.,1912.

Dorfman, Ariel. "Perspectivas y limitaciones de la novela chilena actual" Anales de la Universidad de Chile, No. 140 (1966), pp. 110-167.

..... "Temas y problemas de la narrativa chilena actual". Chile Hoy, Siglo XXI, México, 1970.

.... "Notas para un análisis marxista de la narrativa chilena de los últimos años". Casa de las Américas, Año XII, No. 69 (nov-dic 1971), 65-83.

Drago, Gonzalo. "Cuentos de la generación del 50, de Enrique Lafourcade". Atenea, Año XXXVI, CXXXV, No. 385 (jul-sept 1959), 198-201.

Echevarría, Alfonso. "Dilema entre la libertad y la mediocridad en la literatura chilena actual". Atenea, Año XXXV, CXXXI, No. 380-381 (abr-sept 1958), 276-280.

Edwards, Jorge. "Experiencia personal y creación literaria". Atenea, Año XXXV, CXXXI, No. 380-381 (abr-sept 1958), 280-282.

El movimiento literario de 1842. Compilador Julio Durán Cerda. Santiago, Chile: Editorial Universitaria, 1957.

Encina, Francisco Antonio. "Breve bosque de la literatura histórica chilena". Atenea, Año XXVI, XCV, No. 291-292 (sept-oct 1949), 27-68.

Espinosa, Januario. "La Colonia Tolstoyana". Atenea, Año V, IX, XLII (abril 1928), 166-169.

..... "Augusto d'Halmar y la colonia tolstoyana". Atenea, Año X, XXV, No. 103 (nov 1933), 155-170.

Espinosa, Mario. "Una generación". Atenea, Año XXV, CXXXI, No. 380-381 (abr-sept 1958), 66-77. 
Fein, John. Modernism in Chilean Literature: The Second Period. Durham, N.C.: Duke University Press, 1965.

Ferrero, Mario. "La prosa chilena de medio siglo". Atenea, Año XXXVI, CXXXV, No. 385 (jul-sept 1959), 97-124; Atenea Año XXXVI, CXXXV, No. 386 (oct-dic 1959), 137-153.

Giaconi, Claudio. "Una experiencia literaria". Atenea, Año XXXV, CXXXI, No. 380-381 (abr-sept 1958), 282-289.

Godoy Gallardo, Eduardo. "'Diálogo con Enrique Lafourcade”. Mundo Nuevo, 54 (dic 1970), 65-70.

Guzmán, Nicomedes. "Encuentro emocional con Chile". Atenea, Año XXXV, CXXXI, No. 380-381 (abr-sept 1958), 77-88.

Huneeus Gana, Jorge. Cuadro histórico de la producción intelectual de Chile. Santiago, Chile: Biblioteca de Escritores de Chile, 1910.

Jaén, Didier Tisdel. "Hispanoamérica como problema de la generación romántica en Argentina y Chile”. Disertación, University of Texas, 1965.

Jobet, Julio César. "Notas a propósito de la generación de 1938”. Cultura, 96 (1964), pp. 62-76.

Jones, Willis Knapp. "Editorial Ercilla, Dominant Factor in Latin American Literature". Books Abroad, XI (1936), 173-174.

Kite, Ralph. "Socialist Realism and the Spanish American Novel". Disertación, University of New Mexico, 1968.

Lafourcade, Enrique. "La doctrina del objeto estético". Atenea, Año XXXV, CXXXI, No. 380-381 (abr-sept 1958), 88-97.

.... "Estética y moral de la generación de 1950"'. El Diario Ilustrado, 17 de abril, 1959.

-... “La nueva literatura chilena'”. Cuadernos Americanos, Año XXI, CXXIII (1962), 229-256.

Latcham, Ricardo. “Las ideas del movimiento literario de 1842'”. Atenea, Año XIX, LXVIII, No. 203 (1942), 149-192.

.... "Historia del Criollismo". Anales de la Universidad de Chile, Año CXIII, No. 94 (1954), pp. 3-22.

Latcham, Ricardo; Montenegro, Ernesto; y Vega, Manuel. El Criollismo. Santiago, Chile: Editorial Universitaria, 1956.

Latorre, Mariano. Autobiografía de una vocación: Algunas preguntas que no me han hecho sobre el Criollismo. Santiago, Chile: Editorial Universitaria, 1956.

León, Carlos. “Consideraciones literarias”. Atenea, Año XXXV, CXXXI, No. 380-381 (abr-sept 1958), 97-100.

Leslie, John K. "La polémica del Romanticismo en Chile: Dos artículos desconocidos". Revista Iberoamericana, XVI 1952), 245-254. 
Lillo, Samuel. Espejo del pasado: Memorias literarias. Santiago, Chile: Editorial Nascimento, 1947.

Melfi, Domingo. Indecisión y desengaño de la juventud. Proceso de las generaciones de Chile. Santiago, Chile: Editorial Nascimento, 1935.

.... "Proceso de las generaciones jóvenes de Chile". Atenea, Año XV, XXXI, No. 121 (1935), 51-96.

.... "La generación de Lastarria". Atenea, Año XIV, XXXVII, No. 141 (1937), 235-283.

-... "Estudios de la literatura chilena: El campo en la generación literaria de 1900". Atenea, Año XVI, LXVI, No. 43 (1938), 184-210.

Montenegro, Ernesto. "Aspectos del Criollismo en América". Anales de la Universidad de Chile, Año CXIV, No. 102 (1956), pp. 51-62.

Moretić, Yerko. "Notas para el estudio del realismo socialista en Chile". Principios, órgano del comité central del Partido Comunista de Chile, No. 76 (dic 1960), 28-39.

Moseley, William W. "Origins of the Historial Novel in Chile". Hispania, XLI (1959), 338-342.

Müller, Herbert. "Los escritores jóvenes y los problemas sociales". Atenea, Año XXXV, CXXXI, Nos. 380-381 (abr-sept 1958), 100-105.

Muños Medina, Guillermo. "La generación de 1900 y Augusto G. Thompson". Atenea, Año XII, XXIX, No. 116 (1935), 223-241.

Orrego Luco, Augusto. "El movimiento literario de 1842". Atenea, Año X, XXIV, No. 100 (agosto 1933), 317-350.

Oyarzún, Luis. "Crónica de una generación". Atenea, Año XXXV, CXXXI, Nos. 380-381 (abr-sept 1958), 180-189.

Peralta, Jaime. Cuentistas de la Gereración de 1950. Madrid: Ediciones Insula, 1961.

Pinilla, Norberto, 1842: Panorama y significación del movimiento literario. Santiago, Chile: Ediciones Universidad de Chile, 1942.

.... La generación chilena de 1842. Santiago, Chile: Ediciones Universidad de Chile, 1943.

.... Polémica del Romanticismo en 1842. Buenos Aires: Editorial Americalee, 1943.

Poblete Varas, Hernán. "Novelistas de hoy”. Atenea, Año XXXVII, CXXXIX, No. 389 (jul-sept 1960), 169-181.

Rossel, Milton. "Significación y contenido del Criollismo". Atenea, Año XXXII, CXII, No. 358 (abr-jun 1955), 9-28.

Santana, Francisco. "El movimiento literario de 1842". Atenea, Año XV, LIV, No. 162 (dic 1938), 433-458.

…. "Hombres de 1842". Atenea, Año XIX, LXVIII, No. 203 (mayo 1942), 290 325 . 
.... "La nueva generación de cuentistas chilenos". Atenea, Año XXVI, XCIII, No. 286 (oct 1949), 62-92.

...- La nueva generación de prosistas chilenos. Santiago, Chile: Editorial Nascimento, 1949.

Sánchez Latorre, Luis. Los expedientes de Filebo. Santiago, Chile: Empresa Editora Zig Zag, 1965.

Selva, Pedro. "Historia de las influencias literarias en Chile". Atenea, Año XXIII, LXXXV, Nos. 257-258 (nov-dic 1946), 388-395.

Serrano, Miguel. Ni por mal, ni por tierra...Historia de una generación. Santiago, Chile: Editorial Nascimento, 1950.

Silva Castro, Raúl. "Notas sobre la novela chilena contemporánea". Anales de la Universidad de Chile, Año CXXIV, No. 135 (1967), pp. 254-295.

Skarmeta, Antonio. "La novísima generación: varias características y un límite". American Hispanist, I, 3 (nov 1975), pp. 254-295.

Smith, George Ernest. "The Chilean Literary Scene: 1900". Hispania, XLIII (1960), 552-558.

Teitelboim, Volodia. "La generación del 38 en busca de la realidad chilena". Atenea, Año XXXV, CXXXI, No. 380-381 (abr-sept 1958), 106-131.

Urbistondo, Vicente. El Naturalismo en la novela chilena. Santiago, Chile: Editorial Andrés Bello, 1966.

Valenzuela, Victor. "A New Generation of Chilean Novelists and Short Story Writers". Hispania, XXXVII (1954), 440-442.

Vega, Manuel. "En torno al Criollismo". Anales de la Universidad de Chile, Año CXIII, No. 94 (1954), 23-33.

Vega, Miguel Angel. "Visión panorámica del movimiento literario del 42". Atenea, Año XIX, LXVIII, No. 203 (mayo 1942), 233.239.

Vergara, José Manuel. "Tres actitudes frente a la novela". Atenea, Año XXXV, CXXXI, No. 380-381 (abr-sept 1958), 131-140.

Yankas, Lautaro. "Viaje alrededor del Criollismo". Atenea, Año XXIII, LXXXIV, No. 251 (mayo 1946), 205-209.

-...."Dilucidación del Criollismo". Atenea, Año XXXII, CXXI, No. 360 (jun. 1955), 386-402.

Zamudio Zamora, José. Heinrich Heine en la literatura chilena: Influencia y traducciones. Santiago, Chile: Editorial Andrés Bello, 1958.

.... "Novela histórica y Romanticismo en Chile". Atenea, Año XXXVII, CXXXIX, No. 389 (jul-sept 1960), 255-259.

\section{AUTORES Y NOVELAS}

Alegría, Fernando. "Notas sobre recientes novelas chilenas". Atenea, Año XVIII, LXII, No. 181 (jul 1940), 168-172.

"Manuel Rojas: Trascendentalismo en la novela chilena". Cuadernos Americanos, XVIII, clii (1959), 244-248. 
.... "Nuevos prosistas chilenos". Anales de la Universidad de Chile, Año CXVIII, No. 120 (1960), pp. 175-180.

.... "Lastarria: el precursor". Atenea, Año XXXVII, CXXXIX, No. 389 (julsept 1960), 49-55.

.... "Nuevos prosistas chilenos: notas para integrar las generaciones del $40 \mathrm{y}$ 50". Asomante, XVI, IV (1961), 20-26.

Allen, Martha E. "Dos estilos de novela: Marta Brunet y María Luisa Bombal". Revista Iberoamericana, XVIII, No. 35 (feb-dic 1952), 63-91.

Arce, Magda. Mariano Latorre. Nueva York: Hispanic Institute, 1944.

Arriagada Augier, Julio, y Goldsack, Hugo. Pedro Prado, un clásico de América. Santiago, Chile: Editorial Nascimento, 1952.

-... Augusto D'Halmar: Tres ensayos esenciales y una antologia. Santiago, Chile: Escuela Industrial de Artes Gráficas, 1963.

Arteche, Miguel. "Tres visiones de Carlos Droguett". Cuadernos Hispanoamericanos, 253-254 (enero-febrero 1971), 192-208.

Astorquiza, Eliodoro. "Don Alberto Blest Gana". Atenea, Año XXXVII, CXXXIX, No. 389 (jul-sept 1960), 5-26.

Benavides Lillo, Ricardo. "La fiesta del Rey Acab, novela de Enrique Lafourcade". Atenea, Año XXXVI, CXXXV, No. 385 (jul-sept 1959), 195 198.

Benbow, Jerry L. "Grotesque Elements in Eduardo Barrios". Hispania, LI (1968), 86-91.

Benge, Frances. "Bergson y Pedro Prado". Cuadernos Americanos, Año XXV, CXLVII (jul-agos 1966), 116-123.

Bocaz, Sergio Hernán. "La novelística de José Donoso y su cosmogonía estética a través de dos influencias principales: Marcel Proust y Henry James". Disertación. University of Colorado, 1972.

Bourgeois, Louis C. "Augusto D'Halmar, el Loti hispanoamericano". Hispanófila, 39 (1970), 43-54.

Brown, James W. "El hermano asno from Fioretti Through Freud". Symposium, 25 (1971), 321-332.

Campana, Lorenzo. "Panorama de las letras chilenas en 1959". Atenea, Año XXXVI, CXXXV, No. 386 (oct-dic 1959), 158-175.

Campbell, Margaret. "The Vaporous World of María Luisa Bombal". Hispania, XLIV (1961), 415-419.

Cannizzo, Mary. "Manuel Rojas, Chilean Novelist and Author". Hispania, XLI (1958), 200-201.

Castillo, Homero. "Mariano Latorre". Hispania, XXXVII (1954), 313-316.

-.... “Mariano Latorre y el Criollismo". Hispania, XXXIX (1956), 438-445. 
-... “Tributo a Mariano Latorre". Revista Iberoamericana, XXII, No. 43 (enero-jun 1957), 83-94.

.... "Proyecciones de la crítica y la obra de Mariano Latorre". Atenea, Año XXXVII, CXXXIX, No. 389 (jul-sept 1960), 80-89.

.... " "El mendigo: primer relato novelesco de Chile". Quaderni Iberoamericani, No. 27 (1961), pp. 158-164.

.... "Enrique Araya: Humorista chileno". Hispania, XLIV (1961), 82-88.

Castillo-Feliú, Guillermo Ignacio. "Marginalidad y perspectiva en la obra de José Donoso". Disertación. Michigan State University, 1972.

Correa, Carlos René. "María Luisa Bombal". Atenea, No. 199 (1942), pp. 17-22.

Cortés Larrieu, Norman. "Hijo de ladrón de Manuel Rojas. Tres formas de inconexión en el relato". Anales de la Universidad de Chile, CXVII, No. 120 (1960), 193-202.

Cortínez, Carlos. "Interpretación de El habitante y su esperanza". Revista Iberoamericana, XXIX, Nos. 82, 83 (enero-jun 1973), 159-174.

Cruz-Luis, Adolfo. "iSalve, Droguett! ¡Los que van a matarte saludan!” Casa de las Américas, Año XII, No. 69 (nov-dic 1971), pp. 208-211.

Chapman, Arnold G. "Don Luis Orrego Luco y la vida en Chile". Atenea, Año XXV, XC, No. 278 (1948), 211-232.

Davison, Ned J. "The Significance of 'Del Natural' in the Fiction of Eduardo Barrios". Hispania, XLIV (1961), 27-33.

…. Eduardo Barrios. Nueva York: Twayne Publishers, 1970.

Decker, Donald M. Luis Durand. Nueva York: Twayne Publishers, 1971.

Délano, Luis Enrique. "Una carta sobre d'Halmar". Atenea, Año VI, XIV, No. 66 (agosto 1930), 89-93.

Díaz Arrieta, Hernán [Alone]. Don Alberto Blest Gana. Santiago, Chile: Editorial Nascimento, 1940.

..... "Evocación de Oscar Castro". Atenea, Año XXIV, LXXXVIII, Nos. 269. 270 (nov-dic 1947), 420-428.

..... "Augusto d'Halmar". Atenea, Año XXXVII, CXXXIX, No. 389 (jul-sept 1960), 56-79.

Díaz Márquez, Luis. "Algunas reflexiones sobre los estratos del "narrador" y el "mundo" en la novela Eloy de Carlos Droguett". Horizontes 29 (1971), 5-21.

Drago, Gonzalo. "Vida y obra de Oscar Castro Z". Atenea, Año XXV, LXXXIX, No. 271-272 (enero-feb 1948), 37-50.

.... " "Los Hombres del Hombre de Eduardo Barrios". Atenea, Año XXVIII, C, Nos. 307, 308 (enero-feb 1951), 196-198.

.... “María Nadie de Marta Brunet”. Atenea, Año XXXV, CXXX, No. 379 (enero-marzo 1958), 264-266.

Droguett, Carlos. "Materiales de construcción". Aisthesis: Revista Chilena de Investigaciones Estéticas, No. 3 (1968), pp. 203-225. 
-.... "El escritor y su pasión necesaria". Casa de las Américas, Año XII, No. 68 (sept-oct 1971), 60-68.

Droguett Alfaro, Luis. "Niño de la costa, novela de Juan Negro". Atenea, Año XXXIV, CXXVII, No. 375 (abr-jun 1957), 177-180.

Durán Cerda, Julio. "Marta Brunet, puente de plata hacia el Sur". Anales de la Universidad de Chile, CXIX, No. 124 (1961), 89-94.

Durand, Luis. "Significación de Lastarria". Atenea, Año XIX, LXVIII, No. 203 (mayo 1942), 228-232.

Escudero, Alfonso M. "La actividad literaria chilena en 1924". Atenea, Año II, III, No. 11 (marzo 1925), 66-90; Atenea, Año III, V, No. 21 (Marzo 1926), 65-92.

.... "La actividad literaria chilena en 1926". Atenea, Año IV, VII, No. 35 (jul 1927), 447-479.

Espinosa, Mario. "Cuatro imágenes del Eros en María Carolina Geel". Cuadernos Americanos, Año XXV, CXLVI (1966), 231-239.

Espinoza, Enrique. "Notas sobre Manuel Rojas". Atenea, Año XXXI, CXIV, No. 345 (marzo 1954), 108-121.

-..-. De un lado y otro. Santiago, Chile: Babel, 1956.

Ferrero, Mario. Premios nacionales. Santiago, Chile: Empresa Editora Zig Zag, 1962.

..... Escritores a trasluz. Santiago, Chile: Editorial Universitaria, 1972.

Fogelquist, Donald. "Eduardo Barrios en su etapa actual". Revista Iberoamericana, XVIII, No. 35 (feb-dic 1952), 13-26.

Fuenzalida, Héctor. Esquema y perfiles: notas criticas. Santiago, Chile: Anales de la Universidad de Chile, 1959.

-..-. "Luis Durand y sus novelas". Atenea, Año XXXVII, CXXXIX, No. 389 (jul-sept 1960), 90-102.

Fuenzalida Grandón, Alejandro. Valor histórico de la novela social contemporánea. Santiago, Chile: Imprenta Nacional, 1889.

Gallardo, Andrés. "Vicente Huidobro y las novelas de poetas". Aisthesis: Revista de Investigaciones Estéticas, No. 3 (1968), pp. 95-112.

García, Pablo. "Aporte para la interpretación estética de Juan Godoy”. Atenea, Año XXVI, XCIV, No. 289-290 (1949), 197-202.

García Oldini, Fernando. Doce escritores hasta el año 1925. Santiago, Chile: Editorial Nascimento, 1929.

Geel, María Carolina. Siete escritoras chilenas. Santiago, Chile: Editorial Rapanui, 1953.

Gertel, Zunilda. "Función estructural del 'leit-motiv' en Hijo de ladrón". Revista Hispánica Moderna, XXXV (1969), 363-369.

Goić, Cedomil. “Jemmy Button. Novela por Benjamín Subercaseaux". Atenea, Año XXVIII, CI, No. 312 (jun 1951), 497-501. 
..... "Realismo en Mariano Latorre". Atenea, Año XXXIII, CXXIV, No. 370 (1956), 118-124.

-.-.. "Hijo de ladrón: Libertad y lágrimas". Atenea, Año XXXVII, CXXXIX, No. 389 (jul-sept 1960), 103-113.

..... "La novela chilena actual: Tendencias y generaciones". Anales de la Universidad de Chile, Año CXVIII, No. 119 (1960), 250-258.

..... "La última niebla: Consideraciones en torno a la estructura de la novela contemporánea". Anales de la Universidad de Chile, Año CXXI, No. 128 (1963), pp. 59-83.

…. La novela chilena. Santiago, Chile: Editorial Universitaria, 1968.

Goldemberg, Isaac. "Coronación, de José Donoso o los límites del aislamiento". Mundo Nuevo, 36 (1969), pp. 74-80.

González Vera, José Santos. Cuando era muchacho. Santiago, Chile: Editorial Nascimento, 1951.

.... Algunos. Santiago, Chile: Editorial Nascimento, 1959.

González Zenteno, Luis. "Nicomedes Guzmán, figura representativa de la generación del 38”. Atenea, Año XXXVIII, CXLII, No. 392 (abr-jun 1961), 116-127.

Griffin, Roderic. "The Novels of Augusto d'Halmar: Juana Lucero, La sombra del humo en el espejo, Pasión y muerte del cura Deusto". Disertación, University of Indiana, 1972.

Guzmán, Nicomedes. "Carlos Sepúlveda Leyton, novelista del pueblo". Atenea, Año XVIII, LXIII, No. 189 (marzo 1941), 354-361.

Hamilton, Carlos D. "La novelística de Eduardo Barrios". Cuadernos Americanos, XV, i (1956), 280-292.

Hancock, Joel C. "The Purification of Eduardo Barrios' Sensorial Prose". Hispania, LVI (1973), 51-59.

Hayes de Huneeus, Dorothy. "Recent Literature in Chile". Américas, XI (marzo 1959), 38-41; Américas, XI (sept 1959), 40-43.

Huerta, Eleazar. "El novelista Alberto Romero". Atenea, Año XXXVII, CXXXIX, No. 389 (jul-sept 1960), 114-122.

Hulet, Claude L. "Lafourcade's Roman à Clef". Hispania, XLV (1962), 67-69. Ivelic Kusanovic, Radoslav, y Sepúlveda, Fidel. "Bases críticas para una valoración de la novela chilena". Aisthesis: Revista Chilena de Investigaciones Estéticas, No. 3 (1968), pp. 45-93.

Jobet, Jorge. "Sangre de murciélago, novela de Juan Godoy". Atenea, Año XXXVII, CXXXVII, No. 387 (enero-marzo 1960), 154-160.

Kelley, John R. "The Sentiment of Nature in the Prose Works of Pedro Prado". Disertación, University of Southern California, 1968.

.... "Name Symbolism in Barrios' El hermano asno". Romance Notes, XIII (1971), 48-53. 
Labarca Garat, Gustavo. "Semblanza de Augusto d'Halmar". Atenea, Año XXVII, XCVII, No. 298 (abr 1950), 21-29.

Labarca H., Amanda. "Hijo de ladrón, novela de Manuel Rojas". Atenea, XXVIII, CIII, No. 315-316 (sept-oct 1951), 404-412.

L.A.M. "Coronación, novela de Jose Donoso". Atenea, Año XXXIV, CXXIX, No. 378 (oct-dic 1957), 295-296.

Latcham, Ricardo. Escalpelo. Santiago, Chile: Imprenta de San José, 1925.

..... "Las novelas de Januario Espinosa". Atenea, Año VII, XIV, No. 66 (agosto 1930), 75-79.

..... "Novelistas chilenos de la generación del 40". Estudios Americanos, XLV (1955), 643-673.

....."Blest Gana y la novela realista." Anales de la Universidad de Chile, Año CXVI, No. 112 (1958), pp. 30-46.

..... Carnet critico. Montevideo, Uruguay: Editorial Alfa, 1962.

.... Páginas escogidas. Selección, ordenación y notas de Pedro Lastra y Alfonso Calderón. Santiago, Chile: Editorial Andrés Bello, 1969.

Latorre, Mariano. Memorias y otras confidencias. Selección, prólogo y notas de Alfonso Calderón. Santiago, Chile: Editorial Andrés Bello, 1971.

L.D. "Coirón, novela de Daniel Belmar". Atenea, Año XXVIII, CI, No. 311 (mayo 1951), 250-253.

Lefebvre, Alfredo. "Daniel y los leones dorados por José Manuel Vergara". Atenea, Año XXXIV, CXXVII, No. 374 (enero-marzo 1957), 133-137.

.... "Valores y mensaje de Daniel y los leones dorados". Atenea, Año XXXIV, CXXIX, No. 377 (jul-agosto-sept 1957), 190-203.

Lichtblau, Myron. "Elementos estilísticos de Hijo de ladrón". Humanitas, 5, Monterrey (1964), 323-339.

..... "Ironic Devices in Manuel Rojas' Hijo de ladrón". Symposium, 29 (1965), 214-225.

Loveluck, Juan. "Coirón de Daniel Belmar”. Atenea, Año XXVIII, CI, No. 312 (jun 1951), 491-497.

Lozano, Carlos. "Paralelismo entre Flaubert y Eduardo Barrios". Revista Iberoamericana, XXIV, No. 47 (1959), 105-116.

Lyon, Thomas Edgar. Juan Godoy. Nueva York: Twayne Publishers, 1972.

Martínez Palacio, Javier. "Una gran novela modernista, Alsino". Insula 25 (1970), 15.

Matte Alessandri, Ester. "Siete escritoras de Chile". Anales de la Universidad de Chile, CXVIII, Nos. 121-122 (1959), 134-141.

Meléndez, Luis. "Panorama de los escritores chilenos". Atenea, Año XXXIX, CXLII, No. 392 (1962), 109-115.

Melfi, Domingo. "Panorama literario chileno". Atenea, Año VI, XII, No. 58 loct 1929), 287-296. 
.... "Perspectiva de la novela chilena". Atenea, Año VI, XII, No. 60 (dic 1929), 601-604.

..... "Blest Gana y la sociedad chilena". Atenea, Año X, XXIV, No. 100 (agosto 1933), 162-179.

.... "Luis Orrego Luco, novelista". Atenea, Año XXXVII, CXXXIX, No. 389 (jul-sept 1960), 123-139.

Mengod, Vicente. "El mundo herido de Armando Méndez Carrasco". Atenea, Año XXXII, CXXII, No. 363-364 (sept-oct 1955), 370-373.

Merino Reyes, Luis. "María Luisa Bombal". Atenea, Año XXXVI, CXXXIV, No. 384 (abr-jun 1959), 131-135.

-.-. "Juan Godoy". Atenea, Año XXXVI, CXXXV, No. 385 (jul-sept 1959), 148-151.

"Juan Marín, médico, cuentista, novelista". Revista Interamericana de Bibliografía, XIV (1964), 3-20.

.... “Tres novelistas actuales". Atenea, Año XXXVII, CXXXIX, No. 389 (julsept 1960), 140-152.

Montenegro, Ernesto. Mis contemporáneos: Ensayos biográficos y de crítica literaria. Santiago, Chile: Editorial Universitaria, 1967.

Morales, Leonidas. "La ciudad y el paisaje en la obra de Santiván". Atenea, Año XLIV, CLXVI, No. 417 (jul-sept 1967), 77-91.

Moretić, Yerko. "El Huésped, de Margarita Aguirre". Atenea, Año XXXVI, CXXXIV, No. 384 (abr-jun 1959), 192-195.

.... "Puerto engaño, de Leonardo Espinoza". Atenea, Año XXXVI, CXXXV, No. 385 (jul-sept 1959), 206-208.

.... "Sangre de murciélago, de Juan Godoy". Atenea, Año XXXVI, CXXXV, No. 385 (jul-sept 1959), 201-203.

.... "Eloy, por Carlos Droguett". Atenea, Año XXXVII, CXXXVIII, No. 388 (abr-jun 1960), 255-259.

Morinigo, Mariano. Eduardo Barrios, novelista. Tucumán: Universidad Nacional de Tucumán, 1971.

Núñez, Félix Armando. "Frontera, novela de Luis Durand". Atenea, Año XXVIII, C, No. 307-308 (enero-feb 1951), 173-180.

Opazo, Carlos. "Acerca de una perspectiva humana en Caballo de copas, de Fernando Alegría". Homenaje a Fernando Alegría. Variaciones interpretativas en torno a su obra. Editor Helmy F. Giacoman. New York: L.A. Publishing Company, Inc., 1972.

Orlandi, Julio. "Trayectoria de Joaquín Edwards Bello". Atenea, Año XXXVII, CXXXIX, No. 389 (jul-sept 1960), 153-168.

Orlandi, Julio, y Ramírez, Alejandro. Augusto D'Halmar: Obras, estilo, técnica. Santiago, Chile: Editorial del Pacífico, 1958. 
..-.. Joaquín Edwards Bello: Obras,estilo, técnica. Santiago, Chile: Editorial del Pacífico, 1959.

.... Mariano Latorre: Obras, estilo, técnica. Santiago, Chile: Editorial del Pacifico, 1959.

Ornstein, Jacob. "Breve panorama de la novela chilena reciente". Revista Iberoamericana, XVIII (1954), 339-344.

Orrego Vicuña, Eugenio. “Don Luis Orrego Luco". Atenea, Año XXVI, XCIII, No. 288 (jun 1949), 473-491.

Osorio, Nelson. "Sepúlveda Leyton: Tradición y modernidad". Anales de la Universidad de Chile, 125, Nos. 141-144 (1967), 55-73.

.... "La estructura del narrador y la composición de Caballo de copas". Homenaje a Fernando Alegría. Variaciones interpretativas en torno a su obra. Editor Helmy F. Giacoman. New York: L.A. Publishing Company Inc., 1972.

Osses, Mario. "La sangre y la esperanza, novela de Nicomedes Guzmán”. Atenea, Año XXI, LXXVI, No. 228-229 (jun-jul 1944), 319-323.

..... "Coirón de Daniel Belmar". Atenea, Año XXVIII, CI, No. 312 (jun 1951), 481-482.

Ostaria González, Mauricio. "El monólogo de Eloy". Nueva Narrativa Hispanoamericana, 3, ii (1972), pp. 179-189.

Oyarzún, Luis. El pensamiento de Lastarria. Santiago, Chile: Editorial Jurídica de Chile, 1953.

Pagés Larraya, Antonio. "El vado de la noche de Lautaro Yankas". Atenea, Año XXXIV, CXXVII, No. 375 (abr-jun 1957), 167-170.

Palacios, José María. "El vado de la noche, de Lautaro Yankas". Atenea, Año XXXIII, CXXIV, Nos. 367-368 (enero-feb 1956), 178-182.

Pearson, Lon. "Nicomedes Guzmán: Proletarian Author in Chile's Literary Generation of 1938". Disertación, University of California, Los Angeles, 1973.

Peel, Roger M. "The Narrative Prose of Marta Brunet". Disertación, Yale University, 1967.

Peralta, Jaime. "La novelística de Eduardo Barrios". Cuadernos Hispanoamericanos, LVIII (1964), 357-67.

Petersen, Gerald W. "The Narrative Art of Pedro Prado". Disertación, University of Illinois, 1967.

Phillips, Walter T. "Chilean Customs in Blest Gana's Novels". Hispania, XXVI (1943), 397-406.

Poblete Varas, Hernán. Genio y figura de Alberto Blest Gana. Buenos Aires: Editorial Universitaria, 1968.

Promis Ojeda, José. "El sentido de la existencia en La sangre y la esperanza de Nicomedes Guzmán". Anales de la Universidad de Chile, Año CXXVI, No. 145 (1968), pp. 58-68. 
Ramírez, Manuel D. "Some Notes on the Prose Style of Eduardo Barrios". Romance Notes, IX (1967), 40-48.

Rodríguez Monegal, Emir. “El mundo de José Donoso”. Mundo Nuevo, 12 (jun 1967), pp. 77-85.

Rojas, Manuel. Acerca de la literatura chilena. Santiago, Chile: Imprenta Universitaria, s.f.

-..-. De la poesía a la revolución. Ediciones Ercilla, Santiago, Chile, 1938.

Rojas Piña, Benjamín. "Tres novelas de Emilio Rodríguez Mendoza". Atenea, Año XXXVII, CXL, No. 390 (oct-dic 1960), 157-67.

-... "Bibliografía y cronología de Eduardo Barrios". Boletín del Instituto de Literatura Chilena, Vol. II (oct 1962), pp. 14-24.

...-. "Cifra solitaria, por Juan Godoy". Atenea, Año XL, CL (jul-sept 1963), $221 \cdot 24$.

Rossel, Milton. "El hombre y su psique en las novelas de Eduardo Barrios". Atenea, Año XXXVII, CXXXIX, No. 389 (jul-sept 1960), 182-207.

-... "Reencúentro con Marta Brunet". Atenea, Año XXXVIII, CXLIV, No. 394 (oct-dic 1961), 3-13.

Ruiz, René. “Lukács y la novelística de Fernando Alegría”. Disertación, New York University, 1972.

Russell, Dora Isella. “Marta Brunet”. Cultura Universitaria, 98/99 (enero-jun 1968), 20-24.

Santana, Francisco. Mariano Latorre. Santiago, Chile: Editora Augusto Bello, 1956.

.... "Bosquejo de las novelistas chilenas". Atenea, Año XXXVII, CXXXIX, No. 389 (jul-sept 1960), 208-18.

Sánchez, Porfirio. “Aspectos quijotescos de El niño que enloqueció de amor". Romance Notes, XII (1970), 55-61.

Scott, Robert H. “The Writings of Manuel Rojas”. Disertación, University of Kansas, 1968.

Serros, Robert. "La felicidad vista a través del amor, la soledad y la muerte en la obra literaria de María Luisa Bombal”'. Disertación, University of Southern California, 1969.

Silva Castro, Raúl. "Para la futura novela chilena”. Atenea, Año VII, XIII, No. 64 (jun 1930), 399-411.

..... Retratos literarios. Santiago, Chile: Ediciones Ercilla, 1932.

-..-. Blest Gana y su novela Durante la Reconquista. Santiago, Chile: Imprenta Universitaria, 1934.

.... Panorama de la novela chilena (1843-1953). México: Fondo de Cultura Económica, 1955.

.... "La obra novelística del chileno Alberto Blest Gana". Cuadernos 
Hispanoamericanos, XCI (1967), 224-46.

.... " María Nadie, novela, de Marta Brunet". Atenea, Año XXIV, CXXIX, No. 378 (oct-dic 1957), 258-262.

..... "Sobre Lastarria". Cuadernos Hispanoamericanos, XCVII (1958), 26-44; XCVIII (1958), 167-89.

..... "Mariano Latorre y su novela La paquera". Revista Iberoamericana, XXIV (1959), 297-306.

.... "Blest Gana y su novela La aritmética en el amor". Atenea, Año XXXVII, CXXXIX, No. 389 (jul-sept 1960), 27-47.

.... "Pedro Prado: vida y obra". Revista Hispánica Moderna, XXVI (1960), $1-80$.

.... Historia crítica de la novela chilena. Madrid: Ediciones Cultura Hispánica, 1960.

.... "Manuel Rojas, novelista". Cuadernos Hispanoamericanos, CIII (1960), 519.

.... "El centenario de Martin Rivas". Revista Iberoamericana, XXIX (1963), 139-46.

.... "La obra novelesca de Eduardo Barrios". Cuadernos del congreso por la libertad de la cultura, No. 78 (1963), pp. 76-82.

...-. "Manuel Rojas: Chilean Novelist and Essayist". Books Abroad, XXXVII (1963), 400-02.

..-. "Eduardo Barrios, 1884-1963". Revista Iberoamericana, XXX (1964), 23960.

-.-. "Mujeres en las letras chilenas". Cuadernos del congreso por la libertad de la cultura, No. 94 (1964), pp. 75-80.

-.-.. Pedro Prado (1886-1952). Santiago, Chile: Editorial Andrés Bello, 1965.

Smith, George Ernest. 'D'Halmar, Fantasist". Disertación, Indiana University, 1959.

Solar, Hernán del. "Nuestra literatura de 1960". Atenea, Año XXXVII, CXL, No. 390 (oct-dic 1960), 195-203.

Solar Correa, Eduardo. Escritores de Chile. Santiago, Chile: Imprenta Universitaria, 1932.

Sorel, Andrés. "La nueva novela latinoamericana: I Uruguay, Bolivia, Chile". Cuadernos Hispanoamericanos, LXIV (1965), 221-254.

Sosnowski, Saúl. "El agua, motivo primordial de La última niebla". Cuadernos Hispanoamericanos, No. 277-278 (jul-agosto 1973), pp. 365-74.

Stanford, Alonzo G. "Joaquín Edwards Bello and his En el viejo almendral". Hispania, XXVII (1944), 466-472.

Suárez Miraval, Manuel. "Ránquil: una tendencia de la novelística chilena". Atenea, Año XXIV, LXXXVI, No. 264 (jun 1947), 240-46. 
Swain, James O. Juan Marin-Chilean: The Man and His Writings. Cleveland: Pathway Press, Inc., 1971.

Torres-Ríoseco, Arturo. "Joaquín Edwards Bello". Atenea, Año XVII, LX, No. 178 (abr 1940), 72-108.

-.... "El nuevo estilo en la novela". Revista Iberoamericana, II, No. 5 (1941), $75 \cdot 83$.

-.-. Grandes novelistas de la América Hispana. Berkeley: University of California Press, 1943.

.... "Las novelas de Pedro Prado". Atenea, Año XXXVII, CXXXIX, No. 389 (jul-sept 1960), 219-30.

.... "Nuevas consideraciones sobre la novela chilena". Papeles de Son Armadans, XXXIII (1964), 7-16.

Tull, John F. "El desarmollo de la novela de Marta Brunet". Duquesne Hispanic Review, V, No. 2 (1966), 57-62.

Ulyses. "El Criollismo de Marta Brunet". Atenea, Año XXXII, CXXII, No. 363-364 (sept-oct 1955), 338-41.

Uriarte, Fernando. "Gran señor y rajadiablos por Eduardo Barrios". Atenea, Año XXVII, XCII, No. 283-284 (enero-feb 1948), 138-39.

Uribe Arce, Armando. "Panorama personal de la actual literatura en Chile". Annali di ca' Foscari (Venezia), II, (1963), 155-161.

Uribe Echevarría, Juan. "La paquera, novela postergada de Mariano Latorre". Atenea, Año XXXIII, CXXIV, No. 370 (mayo-jun 1956), 34-53.

Urzúa Aracena, Mario. "Retrato hablado de Carlos Droguett". Mapocho, 18 (1969), pp. 87-91.

Vaise, Emilio. "Casa grande. Estudio sobre algunos tipos y algunas ideas de la novela de Luis Orrego Luco". Anales de la Universidad de Chile, Año CVI, No. 69-72 (1948), 225-38.

Valdés de Undurraga, Adriana. "Las novelistas chilenas: Breve visión histórica y reseña crítica". Aisthesis. Revista Chilena de Investigaciones Estéticas, No. 3 (1968), pp. 113-30.

Valdivieso, Jaime. Un asalto a la tradición. Sepúlveda Leyton: Vida y obra. Santiago, Chile: Editorial Universitaria, 1963.

Valenzuela, Víctor. Cuatro escritores chilenos. Nueva York: Las Americas Publishing Co., 1961.

-... Chilean Society as Seen Through the Novelistic World of Alberto Blest Gana. Santiago, Chile: Talleres de Arancibia Hnos, 1971.

Vásquez-Bigi, Angel. "La verdad sicológica en Eduardo Barrios". Disertación, University of Minnesota, 1962.

.... "Los tres planos de la creación artística en Eduardo Barrios". Revista Iberoamericana, XXXIX (1963), 125-37. 
.... "Los conflictos síquicos y religiosos de El hermano asno". Cuadernos Hispanoamericanos, LXXIII (1968), 456-76; LXXIV (1968), 120-45.

Vial Correa, Elena. "El humor en Joaquín Díaz Garcés". Mapocho, 17 (1968), pp. 39-60.

Vicuña Cifuentes, Julio. He dicho. Santiago, Chile: Editorial Nascimento, 1926. Vidal, Hernán. "El modo narrativo en El hermano asno de Eduardo Barrios". Revista Hispánica Moderna, No. 33 (1967), pp. 241-49.

..... José Donoso: Surrealismo y rebelión de los instintos. Barcelona: Ediciones Aubí, 1972.

Yankas, Lautaro. "Caballo de copas de Fernando Alegría". Atenea, Año XXXV, CXXX, No. 379 (enero-marzo 1958), 251-255.

.... "Cuentistas y novelistas del mar chileno". Atenea, Año XXXVII, CXXXIX, No. 389 (jul-sept 1960), 240-54.

Zamudio Zamora, José. La novela histórica en Chile. Santiago, Chile: Ediciones Flor Nacional, 1949.

\section{ESPACIOS, TEMAS Y MOTIVOS ENLA NOVELA CHILENA.}

Chapman, Arnold G. "Observations on the roto in Chilean Fiction". Hispania, XXXII, (1949), 309-14.

.... "Perspectiva de la novela de la ciudad en Chile". La novela iberoamericana. Editor Arturo Torres-Ríoseco. Albuquerque: University of New Mexico Press, 1951, pp. 191-211.

Chinchón, Osvaldo. "The Sea as a Motif in the Fictional Literature of Chile". Disertación, University of Virginia, 1967.

Díaz Arrieta, Hernán [Alone]. "La alta sociedad y la literatura en Chile". Atenea, Año XX, LXXIII, No. 219 (sept 1943), 234-50.

Durand, Luis. "Visión del campesino chileno". Atenea, Año X, XXIII, No. 98 (jun 1933), 495-503.

.... "La frontera y su interpretación en la literatura chilena". Atenea, Año X, XXIV, No. 101 (sept 1933), 513-532.

Espinosa, Januario. "La clase media en la literatura chilena". Atenea, Año X, XXIV, No. 100(agosto 1933), 276-280.

Illanes Adaro, Graciela. Evolución del sentimiento estético del paisaje en la literatura chilena. Santiago, Chile: Prensas de la Universidad de Chile, 1940.

Latorre, Mariano. "El pueblo chileno en las novelas de Blest Gana". Atenea, Año X, XXIV, No. 100 (agosto 1933), 180-197.

Melfi, Domingo. "El héroe de la clase media en la novela chilena". Atenea, Año VII, XIII, No. 61 (marzo 1930), 65-69.

."La influencia del campo en la novela chilena". Atenea, Año XIII, XXXIV, No. 131 (1936), 171-82. 
Montecinos, Manuel. El mar en la literatura chilena. Santiago, Chile: Editorial del Pacífico, 1958.

Ramírez, Adolf. “The Chilean Novel of Social Protest”. Disertación, University of Wisconsin, 1956.

Salmon, Russell Owen II. "The Roto in Chilean Prose Fiction". Disertación, Columbia University, 1969.

Santana, Francisco. La biografía novelada en Chile. Santiago, Chile: Ediciones Flor Nacional, 1953.

Sender, Ramón. "Sobre la novela rapsódica y la urbe". Revista Iberoamericana, XVII (1953), 269-283.

Silva Castro, Raúl. Paradoja sobre las clases sociales en la literatura. Santiago, Chile: Imprenta Universitaria, s.f.

Torres, Aldo. "El adolescente en la novela chilena". Cuadernos Hispanoamericanos, No. 124 (abr 1960), pp. 80-92.

Uriarte, Fernando. "La novela chilena y la vida intrahistórica”. Atenea, Año XXXVII, CXXXIX, No. 389 (jul-sept 1960), 231-39.

...-. “La novela proletaria en Chile”. Mapocho, IV (1965), 91-103.

Yankas, Lautaro. "Literatura chilena de contenido social". Atenea, Año XVIII, LXIII, No. 188 (1941), 114-32.

Zamudio Zamora,José. "La independencia en la novela histórica chilena". Anales de la Universidad de Chile, Año CXVIII, No. 119 (1960), 236-43.

\section{MATERIAL BIBLIOGRAFICO}

Alarcón, Justo, e Iciar de Sasía, María. Bibliografía literaria de la revista "Hoy". Santiago, Chile: Ediciones de la Biblioteca Nacional, 1970.

Carter, Boyd, G. Historia de la literatura hispanoamericana a través de sus revistas. México: Ediciones de Andrea, 1968 .

Castillo, Homero. "La literatura chilena en los Estados Unidos de América: Ensayo bibliográfico”. Anales de la Universidad de Chile, CXIX, No. 124 (1962), 157-178.

.... La literatura chilena en los Estados Unidos de América. Santiago, Chile: Ediciones de la Biblioteca Nacional, 1963.

Castillo, Homero, y Silva Castro, Raúl. Historia bibliográfica de la novela chilena. México: Ediciones de Andrea, 1961.

Díaz, Miguel Angel. "Revistas literarias chilenas". Atenea, Año XLI, CLIV, No. 404 (abr-jun 1964), 251-53.

Goic', Cedomil. "Bibliografía de la novela chilena del siglo XX". Boletín de Filología de la Universidad de Chile, XIV (1962), 51-168.

J.N. "La revista Hacia". Atenea, Año XXXVI, CXXXV, No. 385 (jul-sept 1959), $188-89$. 
Leavitt, Sturgis E.; Nichols, Madaline; y Rea Spell, Jefferson. Revistas hispanoamericanas: Indice bibliográfico (1843-1935). Santiago, Chile: Fondo Histórico y Bibliográfico José Toribio Medina, 1960.

Lorca, Juan Camilo, y Ruiz-Tagle Gandarillas, Elena. Referencias críticas sobre autores chilenos. Santiago, Chile: Ediciones de la Biblioteca Nacional, 1969.

MacHale, Tomás. Bibliografía de las memorias de grado sobre literatura chilena (1918-1967). Santiago, Chile: Ediciones de la Biblioteca Nacional, 1969.

MacHale, Tomás, y Mendoza, Jaime. Bibliografía general de la revista Estudios (1932-1957). Santiago, Chile: Ediciones de la Biblioteca Nacional, 1969.

Medina, José Toribio. La literatura femenina en Chile. Santiago, Chile, 1923.

Pan American Union. Sección Letras. Diccionario de la Literatura Latinoamericana: Chile. Washington, 1958.

Sánchez, Luis Alberto. Repertorio bibliográfico de la literatura latinoamericana. Tomo III, Chile-Colombia. Santiago, Chile: Editorial Universitaria, 1962.

Silva Arriagada, Luis Ignacio. La novela en Chile. Santiago, Chile: Imprenta y Encuadernación Barcelona, 1910.

Silva Castro, Raúl. Fuentes bibliográficas de la literatura chilena. Tirada aparte de los Anales de la Universidad de Chile. Santiago, Chile: 1933.

Vilches Acuña, Roberto. Las revistas literarias del siglo $X X$. Santiago, Chile: Imprenta Universitaria, 1942.

Yankas, Lautaro. "De la literatura chilena y la crítica". Atenea, Año XLI, CLII, No. 402 (oct-dic 1964), 111-39.

Yurkievich, Saúl. "Rosa naútica, un manifiesto del movimiento de vanguardia chileno". Bulletin de la Faculté des Lettres de Strasbourg, XLVI (1968),64855 . 
